\title{
Bloch's law and a Poisson counting model for simple reaction time to light
}

\author{
JAMES D. HILDRETH \\ West Publishing Co., St. Paul, Minnesota 55165
}

\begin{abstract}
A series of three experiments was conducted with identical design as an earlier series (Hildreth, 1973). Its purpose was (1) to determine whether Bloch's law holds for simple reaction time (RT) to still lower intensity visual stimuli, and (2) to provide data for testing a stochastic generalization of the temporal integration model (TI-ED) reported earlier. RT means were found to agree with Bloch's law for durations below $48 \mathrm{msec}$. By a statistical test, Bloch's law was shown to hold for both means and standard deviations below about $65 \mathrm{msec}$. Latency statistics-means and standard deviations-were predicted by a Poisson process counting model. This model assumes that a number of identical, parallel Poisson processes, activated by light, with pulse interarrival times decreasing with light intensity, trigger light detection when a critical number of pulses arrive at a counting center. For the intensities investigated, both the estimated number of Poisson processes and critical number of pulses required for detection range between 8 and 13. The model predicts the Broca-Sulzer effect for mean RTs which is observed in several of these experiments.
\end{abstract}

At least two reports, Bruder and Kietzman (1973) and Hildreth (1973), have provided evidence that Bloch's law (BL) holds for RTs for durations less than $16 \mathrm{msec}$. It was observed in the former to hold when a narrow range of intensities $(L$ to $L / 4)$ was used within a given experiment. This is a report of further experimentation with lower light intensities, testing the applicability of BL to RTs to thresholdlevel light stimuli.

Secondly, this report proposes a model, the Poisson counting model, of a general class of models first proposed by Luce and Green (1972). As a stochastic generalization of the temporal integration - exponential decay model (TI-ED) presented in Hildreth (1973), the Poisson counting model (1) provides a mechanism for predicting RT means, variances, and detection probabilities; (2) accounts for contextual effects observed in mean RTs: the increase by a constant amount of means to identical stimuli when included in a lower intensity stimulus set; and (3) predicts the Broca-Sulzer effect for RT means, which was observed most clearly in the lowest intensity experiments.

\section{METHOD}

\section{Procedure}

The purpose of these three experiments was to investigate RT statistics for near-threshold light intensities.

Work on this project was supported in part by a research grant from the University of Wisconsin-Eau Claire. The author thanks John I. Yellott, Jr., for his helpful advice and David LaBerge for the use of his laboratory at the University of Minnesota. The author's address is: West Publishing Co., 50 West Kellogg Blvd., P.O. Box 3526, St. Paul, Minnesota 55165.
Each experimental session consisted of a sequence of simple RT trials, $25 \%$ of which were catch (no stimulus) trials. Over the remaining $75 \%$ of the trials, stimulus duration and intensity varied randomly, as described below. Altogether, there were three experiments, corresponding to three different ranges of intensity. Except for differences in luminance and duration values, these experiments were identical in procedure, and the same two subjects participated in all three.

The order in which the experiments for both subjects were run was $4,5,6$. The subjects were extensively practiced before each experiment with new luminance parameters. About six 1-h sessions were typically required to reach asymptotic performance for a single experiment, after which six 1-h data collection sessions were conducted for each. No RTs were discarded from any experiment, although it was necessary to discard some complete day's results due to equipment failure or subject fatigue. ${ }^{1}$ In each such case, the experimental condition was rerun on a subsequent day.

\section{Stimuli}

Stimuli were produced with two fluorescent lamps (GE F8T5 Daylight) in each of three fields of a Scientific Prototype tachistoscope. Each field provided one of the intensity levels. The circuits produced nearly rectangular stimuli, as confirmed by photographs of the electrical output of a photocell. Stimulus duration was controlled by a small computer (CDC 160A) and was tested to an accuracy of within $1 \mathrm{msec}$ at the electronic gate of the tachistoscope. Durations used in the experiments varied from 4 to 256 (see Figures 2 and 3). Stimulus luminance was controlled by neutral density filters calibrated with a Photovolt photometer (520ME). The stimulus fields were congruent, circular, subtended $1.6^{\circ}$, and appeared white when transilluminated by the lamp. A dim red dot, $1^{\circ}$ below the stimulus, served as a fixation point. The subject, dark-adapted for $1 / 2 \mathrm{~h}$, sat in a dark room and viewed binocularly. Head position was controlled by a rubber viewport. The response button was a sensitive microswitch mounted vertically below a handrest and closed by pulling action.

\section{Instructions}

Instructions to the subject were to respond as quickly as possible to the light flashes while maintaining a false alarm (FA) rate of less than $4 \%$. 
Trial Event Sequence

Duration (msec)

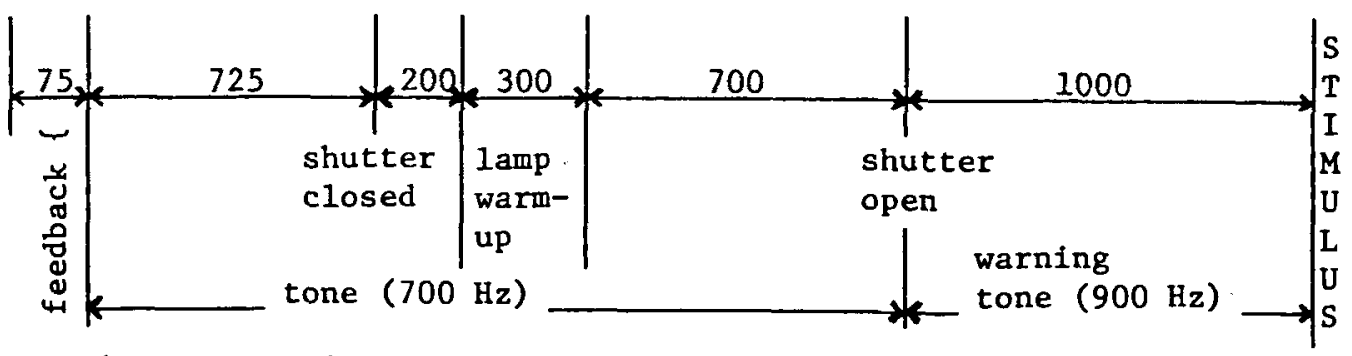

(tones have $25 \mathrm{msec}$ rise and decay time controlled by electronic switch)

Contingent Feedback and Sequencing Control:

\begin{tabular}{|c|c|c|c|}
\hline & & $\begin{array}{l}\text { response } \\
\text { within } \\
1 \text { second }\end{array}$ & $\begin{array}{l}\text { no response } \\
\text { within } \\
1 \text { second }\end{array}$ \\
\hline$(75 \%)$ & $\begin{array}{l}\text { light } \\
\text { stimulus }\end{array}$ & $\begin{array}{l}\text { continue } \\
\text { to next } \\
\text { trial }\end{array}$ & $\begin{array}{l}\text { MISS } \\
\text { (white noise } \\
\text { feedback) \& } \\
\text { cont. to next trial }\end{array}$ \\
\hline$(25 \%)$ & $\begin{array}{l}\text { catch } \\
\text { trial }\end{array}$ & $\begin{array}{l}\text { FALSE ALARM } \\
\text { (speech noise } \\
\text { feedback) \& } \\
\text { cont. to next } \\
\text { trial }\end{array}$ & $\begin{array}{l}\text { continue } \\
\text { to next } \\
\text { trial (after } \\
1 \text { second) }\end{array}$ \\
\hline
\end{tabular}

Figure 1

\section{Event Sequence}

The content and duration of fixed foreperiods and stimulus periods, stimulus randomization and selection, latency measurement and recording, and conditional feedback were controlled by the computer. The trial event sequence, contingent feedback, and sequencing control are shown in Figure 1. This sequence applied on every trial. Because of the critical importance of maintaining equal stimulus characteristics among fields, each trial contained a special bulb warm-up period of $300 \mathrm{msec}$ in all three fields. The resulting flash was masked from the subject's view by a shutter. The warm-up had the effect of minimizing sequential dependencies in latencies due to lamp temperature.

\section{Subjects}

The author served as Subject 1 (S1). A paid male college student served as Subject 2 (S2). Both subjects served in all three experiments.

\section{Experimental Design}

Three experiments were conducted. These were identical in procedure except for luminance and duration values used; luminances are shown in Table 1 by experiment number. Note that the lowest of three intensity values for Experiment $j$ becomes the highest of the three intensities of Experiment $j+1(j=4,5)$. This affords a test of replicability for these luminances and allows investigation of the effect of the context within which a stimulus appears (as the brightest or dimmest of the set).

Each experiment consisted of 6 experimental sessions. Essentially, each experimental session consisted of a sequence of simple RT trials about $3.5 \mathrm{msec}$ apart, with rest periods between each block (32). Within each block, $25 \%$ (8) were catch trials (no stimulus). Over the remaining $75 \%$ (24) trials, stimulus duration and luminance varied randomly; permutations of the 32 stimuli ( $3 \times 8$ luminance-duration combinations plus 8 catch trials) were selected randomly and presented to the subject. Altogether,

Table 1

Luminances by Experiment Number

\begin{tabular}{ccccccc}
\hline Experiment & \multicolumn{3}{c}{ Luminance (fL) } & \multicolumn{2}{c}{ Luminances in Terms of L $=.6 \mathrm{fL}$} \\
\hline 4 & .009375 & .00469 & .002344 & $\mathrm{~L} \times 2^{-6}$ & $\mathrm{~L} \times 2^{-7}$ & $\mathrm{~L} \times 2^{-8}$ \\
5 & .002344 & .00117 & .000586 & $\mathrm{~L} \times 2^{-8}$ & $\mathrm{~L} \times 2^{-9}$ & $\mathrm{~L} \times 2^{-10}$ \\
6 & .000586 & .000293 & .000146 & $\mathrm{~L} \times 2^{-10}$ & $\mathrm{~L} \times 2^{-11}$ & $\mathrm{~L} \times 2^{-12}$ \\
\hline
\end{tabular}


within each session there were 360 stimulus trials and 120 catch trials, or 15 observed RTs for each of the 24 data points (means) per session. Each experiment of 6 sessions therefore consisted of 2,160 observed RTs from stimulus trials and 720 catch trials, with each mean RT data point an average of 90 RTs. To determine stimulus intensity, the three stimulus fields were first equated for intensity to $15 \mathrm{fL}^{2}$ and the neutral density filters inserted into fields of the tachistoscope. Within each session, one-third (6) of the blocks of 32 trials each were run with a given filter-field assignment to control for a possible effect of differences in stimulus characteristics of the three fields, with the first block of each discarded as practice.

It was pointed out that for the lowest intensities, catch trials and misses were confounded so that, in effect, Experiment 6 had more "catch trials" than Experiment 4, possibly increasing resultant $\mathrm{RTs}$. The only precaution in this regard was the contingent feedback: misses were rewarded by white noise 1 sec after the stimulus was presented, whereas correct identification of "no stimulus" 'was rewarded with no feedback (see Figure 1).

\section{EXPERIMENTAL RESULTS}

Mean RTs as a function of duration and intensity are shown in Figures 2 and 3. Each data point represents an average of up to 90 RTs. Open circles represent mean RTs with fewer than 90 observations due to misses. Arrows are drawn between means to stimuli of equal energy. BL predicts they should be horizontal. It appears that BL is not upheld for means to stimuli over about $48 \mathrm{msec}$. A statistical test of the goodness of fit of BL was applied, however, to both means and standard deviations. As reported by Bernstein, Futch, and Schurman (1973), variation within data points to stimuli of equal energy was compared to variation within points to stimuli of equal intensity, using the $F$ statistic. After points beyond the estimated critical duration were excluded, the test showed BL fitted both means and standard deviations of Experiments 4 and 5, but not of Experiment 6. Table 2 shows significance levels and $F$ statistics obtained by experiment number. Estimated critical durations for Experiment 5 range up to $68 \mathrm{msec}$ for the lowest intensity, supporting the conclusion that BL holds for critical durations below that point.

One puzzling aspect of these results also occurred in the earlier experiments (1-3): The same stimulus gave rise to different mean RTs, depending on whether they were the highest or lowest in the experiment. In the context of brighter stimuli: the same stimulus produced faster responses than when in the context of dimmer stimuli. One possible explanation is that this latency difference may be due to dark adaptation, since less adaptation was possible for higher intensity experiments due to trial-to-trial effects. Some evidence exists that visual latency increases with the amount of dark adaptation (Standing, Dodwell, \& Lang, 1968). Another possibility is "expectancy": Since flashes are generally perceived later in the lower intensity experiments, the subject may expect to see them later, causing a delayed RT in those cases. The model to be described below accounts for this latency difference both by changes in the subject's criterion for detection and by changes in the residual, nonperceptual component of RT.

False alarms were consistently below $4 \%$, and were typically less than $2 \%$. Misses were, however, a more significant factor than in earlier experiments. Open

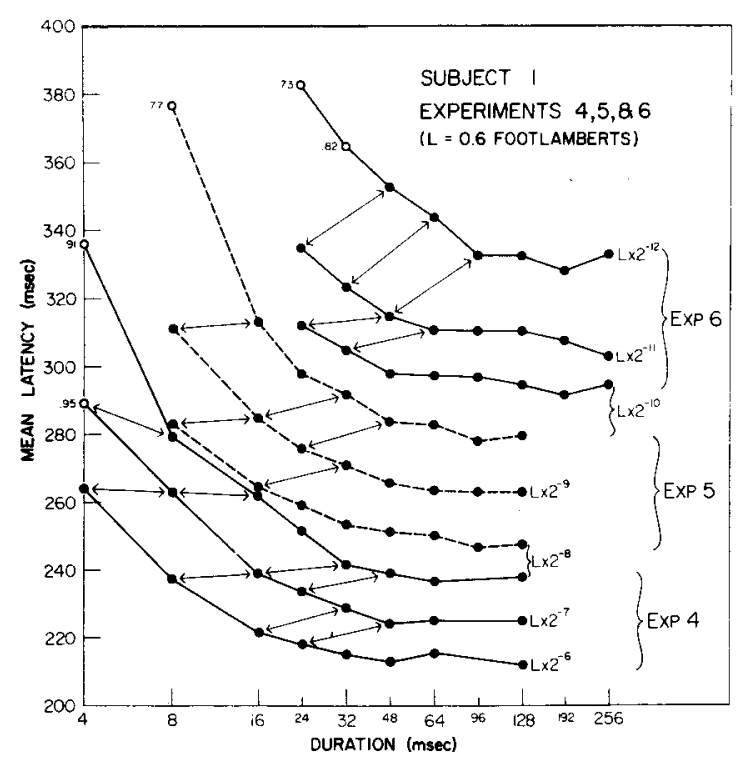

Figure 2. The lines, solid, dashed, and solid, represent mean RTs for both subjects for Experiments 4, 5, and 6, respectively. Luminance parameters on the right, which decrease successively by a factor of a half, may be converted to footlamberts by using the value $L=.6 \mathrm{fL}$. Open circles represent mean RTs with miss proportions greater than .05; the proportion of hits accompanies each such point. Arrows are drawn between points representing equal stimulus energy (luminance by duration product); Bloch's law holds to the extent that they are horizontal.

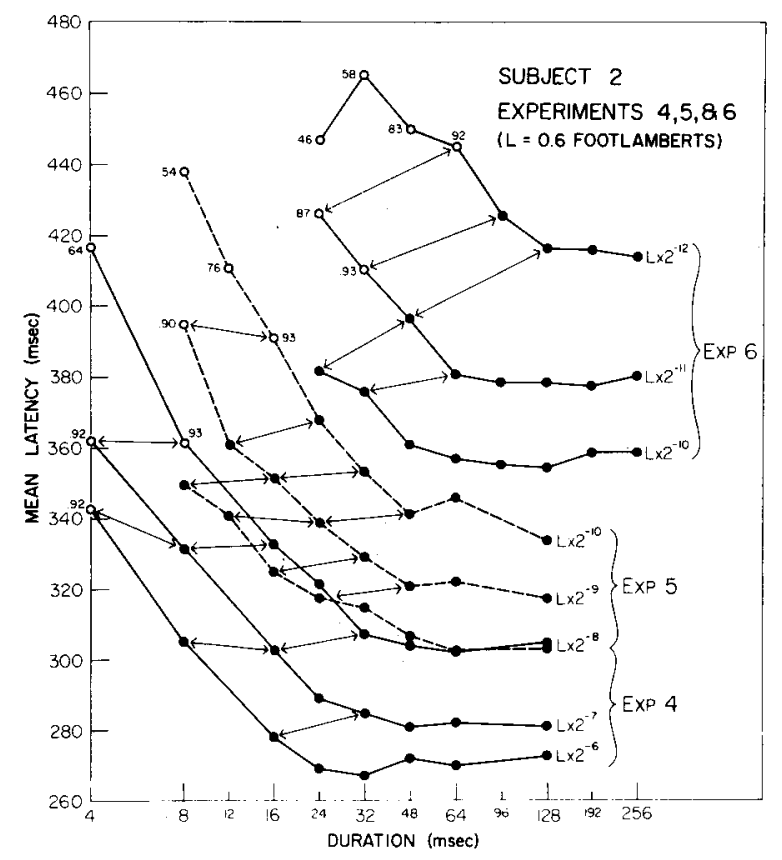

Figure 3. See caption for Figure 2. 
Table 2

Significance of Bloch's Law

\begin{tabular}{|c|c|c|c|c|c|}
\hline \multirow{2}{*}{$\begin{array}{c}\text { Experi- } \\
\text { ment }\end{array}$} & \multirow[b]{2}{*}{ df } & \multicolumn{2}{|c|}{ Mean } & \multicolumn{2}{|c|}{ Standard Deviation } \\
\hline & & $\mathbf{F}$ & $\mathrm{p}^{*}$ & $\mathbf{F}$ & p* \\
\hline \multicolumn{6}{|c|}{ Subject 1} \\
\hline 4 & 12,9 & 20.95 & .001 & 6.91 & .005 \\
\hline 5 & 11,8 & 5.23 & .01 & 6.09 & .01 \\
\hline 6 & 5,4 & .84 & n.s. & 2.53 & n.s. \\
\hline \multicolumn{6}{|c|}{ Subject 2} \\
\hline 4 & 10,8 & 14.60 & .001 & 6.63 & .01 \\
\hline 5 & 14,10 & 14.80 & .001 & 19.51 & .001 \\
\hline 6 & 8,6 & .81 & n.s. & 2.01 & n.s. \\
\hline
\end{tabular}

$" p<$ value given; n.s. = nonsignificant .

circles in Figures 2 and 3 indicate over 3\% misses, and are found as expected to the lowest energy stimuli. The standard deviations of both subjects are shown in Figures 4 and 5. For clarity, the SD scale is displaced upward by $20 \mathrm{msec}$ for each successive experiment. SDs regularly decrease with stimulus duration and intensity, and it is of interest whether BL holds for these SDs. Although it is not clear by inspection, when the Bernstein statistical test is applied to them, the same result is obtained as for means: BL holds for SDs of Experiments 4 and 5, but not Experiment 6 (see Table 2).

\section{POISSON COUNTING MODEL}

\section{Assumptions}

(1) The total human reaction time to a light stimulus contains a component that is independent of the nature of the stimulus itself. Often considered "motor response time," it may be statistically separated out by its independence. We denote this residual component as a random variable, $T_{r}$.

(2) The remaining reaction time, whether considered perceptual only or also involving some decision process, may depend on stimulus characteristics. Of interest to us are the variables of intensity and duration as they affect this time. If $d$ represents duration and $\ell$ represents luminance, then we denote this component $T_{d, \ell}$. Thus, if $R T(d, \ell)$ denotes reaction time,

$$
\mathrm{T}_{\mathrm{d}, \ell}=\mathrm{RT}(\mathrm{d}, \ell)-\mathrm{T}_{\mathrm{r}} .
$$

(3) The subject's criterion for the event of detection is assumed fixed for each stimulus set. That is, he is capable of varying this criterion, but it must be fixed in advance of presentation of a stimulus. If uncertainty exists as to which of a set of stimuli will be presented, the subject must adopt a fixed criterion for the whole stimulus set. He might do this through learning payoffs for accurate detections, false alarms, misses, and accurate detections of no-stimulus. Interesting as these considerations are, we have attempted to eliminate their effects so far as possible by extended practice with each experimental condition, and by randomly selecting from a set of stimuli within each experiment. Thus, for each experiment, the subject is expected to gradually adopt a fixed criterion, $K$, which will not significantly affect the variance of $T_{d, \ell}$. We may, however, expect adoption of different criteria (K) for detection under different experimental conditions which are known in advance.

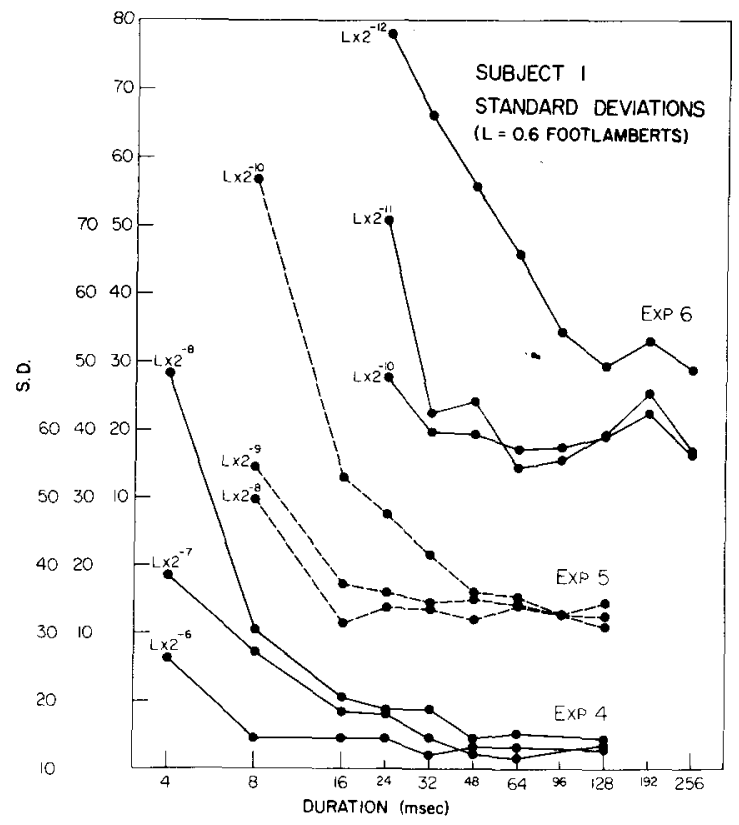

Figure 4. Standard deviations for Experiments 4, 5, and 6 are shown by solid dots connected by solid, dashed, and solid lines, respectively. Luminance parameters are provided on the left. The SD scale on the ordinate is increased by $20 \mathrm{msec}$ for each experiment for clarity.

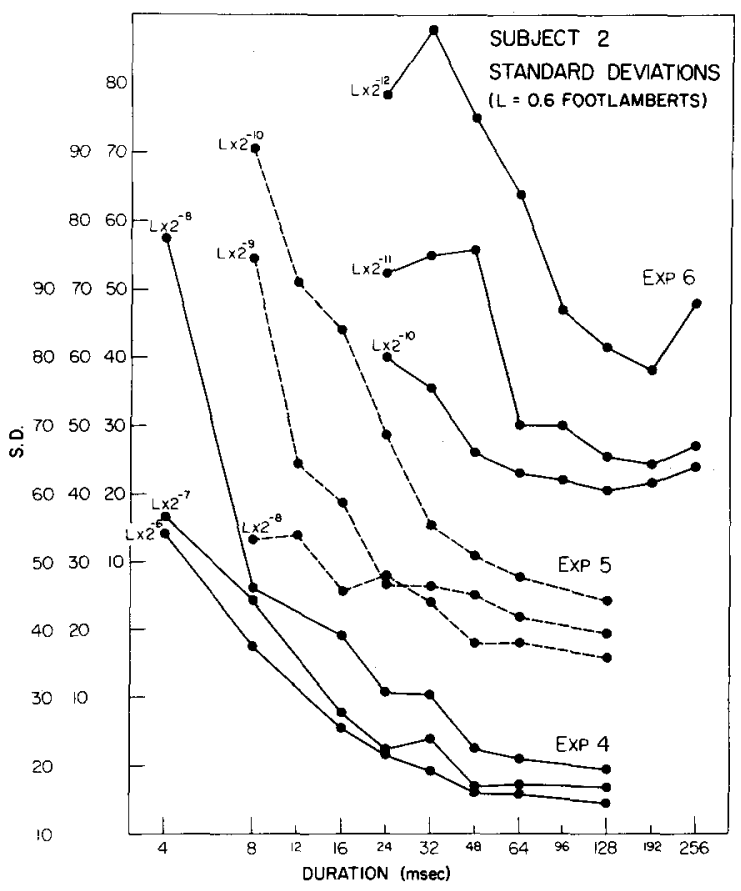

Figure 5. See caption for Figure 4. 
(4) (a) The output of the visual system is a set of sequences of "neural pulses" on a number of parallel channels.

(b) The duration of each pulse is negligible.

(c) Interarrival times (IATs) on each channel are independent random variables.

(d) IATs have a common distribution with expected value which is a strictly monotonic decreasing function of light intensity.

(e) For a given light intensity, $\ell$, these stochastic processes are Poisson processes with the same intensity parameter, $r_{\ell}$. That is, IATs are independent, identically distributed exponentials with mean $1 / r_{\ell}$.

(5) A decision rule is adopted by the subject whereby the visual system triggers a "detect" response after K pulses have been observed (cf. Assumption 3).

(6) (a) The number of parallel Poisson processes at any time is determined by a renewal process with a rate depending on light intensity. The number of processes resulting from this renewal process, $\mathrm{N}_{\boldsymbol{l}}$, is strictly a function of light intensity, $\ell$. This is a simplifying assumption, since it implies that $\mathrm{N}_{\ell}$ processes will be activated, independent of how short a time the light flash is presented. From the results of parameter estimation, described below, it became apparent that a better fit of the model could be obtained at the cost of introducing another parameter representing the rise of $\mathrm{N}$ to a maximum value for very short durations and intensities. However, in the following, we assume that the rise of $\mathrm{N}$ to its maximum value, $N_{\ell}$, occurs practically instantaneously; hence the notation $\mathrm{N}_{\ell}$.

(b) A sudden reduction in light intensity during the detection process, e.g., light offset, results in a lower renewal rate and smaller number of active channels, $\mathrm{N}_{\ell}$. For light offset, we assume $\mathrm{N}_{\ell}=0$ from the background illumination of our experiments. This is yet another simplifying assumption, since we might be able to account for light adaptation effects by introducing a nonzero parameter here. ${ }^{3}$ However, it is the renewal rate that changes with light intensity, and each of the original $\mathrm{N}_{\ell}$ Poisson processes are left with exactly one more pulse to deliver to the detection center. Therefore, the number of processes still active at any point in time will decay exponentially, on the average, after light offset.

\section{Model Statistics}

The statistics of detection times are then a straightforward matter of calculation and curve fitting with parameter estimation. Individual detection times are "waiting times," denoted $\mathrm{W}_{\mathrm{k}, \ell}$, the time required for the Kth pulse to arrive at the detection center for intensity $\ell$. Let $X_{t, \ell}$ denote the number of pulses received by time $t$. Then, at the instant of detection, $\mathrm{t}, \mathrm{X}_{\mathrm{t}, \ell}=\mathrm{K}$ and $\mathrm{t}=\mathrm{W}_{\mathrm{k}, \ell}$. Therefore, $\mathrm{P}\left(\mathrm{W}_{\mathrm{k}, \ell} \leqslant \mathrm{t}\right)$ $=\mathrm{P}\left(\mathrm{X}_{\mathrm{t}, \ell} \geqslant \mathrm{K}\right)$. Three cases may occur in the relation between $\mathrm{W}_{\mathrm{k}, \ell}$ and $\mathrm{d}$, light duration, for any stimulus event:

(1) Detection at or before light offset $\left(W_{k, l} \leqslant d\right)$. This is the simplest case, since the number of Poisson processes is constant and equal to $N_{l}$. The waiting time, $W_{k, \ell}$, is a random variable with gamma distribution of order $\mathrm{K}$ with pdf

$$
f_{k, \ell}(t)=\frac{1}{(K-1) !} N_{\ell} r_{\ell}\left(N_{\ell} r_{\ell} t\right)^{K-1} e^{-N_{\ell} r_{\ell} t}\left(N_{\ell} r_{\ell} t>0\right),
$$

where $1 / r_{l}$ is the decay constant, the common mean interpulse time for a single Poisson process.

(2) Detection after light offset $\left(W_{k, l}>d\right)$. Because the renewal process ends at light offset, the number of processes, $N_{l}$, decrease gradually to zero.

(3) Failure of detection $\left(W_{k, l}=\infty\right)$. Under weak stimulus conditions, $K$ pulses may never arrive within the observing period (the time from warning tone offset to the beginning of the next trial-cf. Figure 1), so no detection occurs.

\section{Expectations}

Conditioning on the event $\mathrm{w}_{\mathrm{k}, \ell} \leqslant \mathrm{d}$, detection before light offset,

$$
E\left(W_{k, \ell} \mid W_{k, \ell} \leqslant d\right)=\frac{1}{P\left(W_{k, \ell} \leqslant d\right)} \int_{0}^{d} \mathrm{tf}_{k, \ell}(t) d t,
$$

where $f_{k, l}(t)$ is as defined in Equation 2. This conditional expectation is of a gamma distribution truncated at $d$. For $d=\infty, E\left(W_{k, \ell}\right)=K /\left(N_{\ell} r_{\ell}\right)$.

The conditional expectation of the waiting time for the event "detection after light offset" is computed differently: After the time of light offset (d), the remaining expected waiting time depends on how many pulses $\left(X_{d, \ell}\right)$ have been received by time $d$. Here the assumption is that exactly one pulse resides in each Poisson process after light offset. Clearly, if $X_{d, \ell}<K-N_{\ell}$, i.e., $X_{d, \ell}+N_{\ell}<K$, then no detection is possible, since even with $N_{l}$ more pulses, the critical number of pulses $(\mathrm{K})$ will still not be reached. Also, if $X_{d, \ell} \geqslant K$, detection will occur before or at light offset, so Equation 3 would apply.

Therefore, using the fact that IATs after light offset are independent exponential random variables with successive mean values, $1 /\left(\mathrm{N}_{\ell} \mathrm{r}_{\ell}\right), 1 /\left[\left(\mathrm{N}_{\ell}-1\right) \mathrm{r}_{\ell}\right]$, $\ldots 1 / r_{l}$,

$$
\begin{gathered}
E\left(W_{k, \ell} \mid d<W_{k, \ell}<\infty\right) \\
=d+\sum_{i=K-N_{\ell}}^{K-1} P\left(X_{d, \ell}=i\right) \sum_{j=1}^{K-i} \frac{1}{\left(N_{\ell}-j+1\right) r_{\ell}} \\
\left(X_{d, \ell}=0,1, \ldots\right) .
\end{gathered}
$$


Finally, since misses have no reaction time associated with them, and none are included in the set of reaction times, the above two expectations combined yield the expected detection time

$$
\begin{aligned}
\mathrm{E}\left(\mathrm{W}_{\mathrm{k}, \ell} \mid \text { detection }\right) \\
=\mathrm{E}\left(\mathrm{W}_{\mathrm{k}, \ell} \mid \mathrm{W}_{\mathrm{k}, \ell}<\infty\right) \\
=\mathrm{P}\left(\mathrm{W}_{\mathrm{k}, \ell} \leqslant \mathrm{d}\right) \mathrm{E}\left(\mathrm{W}_{\mathrm{k}, \ell} \mid \mathrm{W}_{\mathrm{k}, \ell} \leqslant \mathrm{d}\right) \\
\quad+\mathrm{P}\left(\mathrm{d}<\mathrm{W}_{\mathrm{k}, \ell}<\infty\right) \mathrm{E}\left(\mathrm{W}_{\mathrm{k}, \ell} \mid \mathrm{d}<\mathrm{W}_{\mathrm{k}, \ell}<\infty\right) .
\end{aligned}
$$

Equations 2-4 combined with Equation 5 yield expectations for the visual component of reaction time, $\mathrm{Td}$, as a function of model parameters.

\section{Variance}

The total variance of the waiting time, $\mathrm{W}_{\mathrm{k}}$, will be the sum

$$
\operatorname{Var}\left(\mathbf{W}_{\mathrm{k}, \ell}\right)=\operatorname{Var}\left[\mathrm{E}\left(\mathrm{W}_{\mathrm{k}, \ell}\right)\right]+\mathrm{EVar}\left(\mathrm{W}_{\mathrm{k}, \ell}\right) .
$$

Starting first with the latter term, and again conditioning on whether detection occurs before or after offset,

$$
\begin{aligned}
& \operatorname{EVar}\left(\mathrm{W}_{\mathrm{k}, \ell} \mid \mathrm{W}_{\mathrm{k}, \ell} \leqslant \mathrm{d}\right) \\
& \quad=\frac{1}{\mathrm{P}\left(\mathrm{W}_{\mathrm{k}, \ell} \leqslant \mathrm{d}\right)}\left\{\int_{0}^{\mathrm{d}} \mathrm{t}^{2} \mathrm{f}_{\mathrm{k}, \ell}(\mathrm{t}) \mathrm{dt}-\left[\int_{0}^{\mathrm{d}} \mathrm{tf}_{\mathrm{k}, \ell}(\mathrm{t}) \mathrm{dt}\right]^{2}\right\}
\end{aligned}
$$

$$
\begin{aligned}
& E \operatorname{Var}\left(W_{k, \ell} \mid d<W_{k, \ell}<\infty\right) \\
& =\sum_{i=k-N_{\ell}}^{k-1} P\left(X_{d, \ell}=i\right) \sum_{j=1}^{k-i} \frac{1}{\left(N_{\ell}-j+1\right)^{2} r_{\ell}{ }^{2}},
\end{aligned}
$$

since $d$ is a constant. Combining Equations $7 a$ and $7 b$,

$$
\begin{aligned}
& \operatorname{EVar}\left(\mathrm{W}_{\mathrm{k}, \ell}\right) \\
& =\operatorname{P}\left(\mathrm{W}_{\mathrm{k}, \ell} \leqslant \mathrm{d}\right) E \operatorname{Var}\left(\mathrm{W}_{\mathrm{k}, \ell} \mid \mathrm{W}_{\mathrm{k}, \ell} \leqslant \mathrm{d}\right) \\
& \quad+\mathrm{P}\left(\mathrm{d}<\mathrm{W}_{\mathrm{k}, \ell}<\infty\right) E \operatorname{Var}\left(\mathrm{W}_{\mathrm{k}, \ell} \mid \mathrm{d}<\mathrm{W}_{\mathrm{k}, \ell}<\infty\right) .
\end{aligned}
$$

Secondly, for the former term of Equation 6,

$$
\begin{aligned}
& \operatorname{VarE}\left(\mathrm{W}_{\mathrm{k}, \ell}\right) \\
& =\mathrm{P}\left(\mathrm{W}_{\mathrm{k}, \ell} \leqslant \mathrm{d}\right)\left[\mathrm{E}\left(\mathrm{W}_{\mathrm{k}, \ell} \mid \mathrm{W}_{\mathrm{k}, \ell} \leqslant \mathrm{d}\right)-\mathrm{E}\left(\mathrm{W}_{\mathrm{k}, \ell}\right)\right]^{2} \\
& \quad+\mathrm{P}\left(\mathrm{d}<\mathrm{W}_{\mathrm{k}, \ell}<\infty\right)\left[\mathrm{E}\left(\mathrm{W}_{\mathrm{k}, \ell} \mid \mathrm{d}<\mathrm{W}_{\mathrm{k}, \ell}<\infty\right)\right. \\
& \left.\quad-\mathrm{E}\left(\mathrm{W}_{\mathrm{k}, \ell}\right)\right]^{2}
\end{aligned}
$$

Finally, combining Equations 8 and 9 yields the desired total variance estimate of $T_{d}$, the visual component of $\mathrm{RT}$, as a function of model parameters. Note that

$$
\begin{aligned}
\lim _{d \rightarrow \infty} \operatorname{Var}\left(W_{k, \ell}\right) & =\int_{0}^{\infty} t^{2} f_{k, \ell}(t) d t-\left[\int_{0}^{\infty} t_{k, \ell}(t) d t\right]^{2} \\
& =\frac{k(k+1)}{\left(N_{\ell} r\right)^{2}}-\left(\frac{k}{N_{\ell} r}\right)^{2}=\frac{k}{\left(N_{\ell} r\right)^{2}}
\end{aligned}
$$

The total E(RT) and $\operatorname{Var}(\mathrm{RT})$ estimates are obtained by the assumption of independence of the perceptual and nonperceptual components. Estimates of constants ETr and VarTr were added to $E\left(T_{d}\right)$ and $\operatorname{Var}\left(\mathrm{T}_{\mathrm{d}}\right)$, respectively, to obtain $\mathrm{RT}$ statistics predictions.

\section{Parameter Estimation}

The model generates RT statistics-means and standard deviations-and miss probabilities as a function of: $N_{\ell}$, the number of identical Poisson processes generated by a light stimulus of intensity $\ell$, and $1 / r_{\ell}$, the expected interarrival time between pulses for a single Poisson process generated by a light stimulus of intensity $\ell$.

The following are controlled by the subject and assumed fixed within each experiment: $\mathrm{K}$-the number of pulses required for detection-criterion; $\mathrm{ETr}$ - the expected residual $\mathrm{RT}$; and $\mathrm{SDr}$ - the standard deviation of residual $R T$.

In estimation of parameters $N_{l}$ and $1 / r_{\ell}$, the fact that the identical stimulus was used in successive experiments - the dimmest of a set of three in one experiment was the brightest of a set in the next experiment-was used to advantage: $N_{l}$ and $1 / r_{l}$ must have the same values for RTs to the same stimulus in each pair of experiments. Thus cross-experimental data was used to initially estimate these parameters via a least squares procedure. The subjectcontrolled parameters were then estimated from within-experiment data using the same least squares method. Finally, the constraints on the parameters were used simultaneously for their estimation: (1) $\mathrm{N}_{\ell}$ and $1 / r_{\ell}$ were required to be identical for the same stimulus even when it appeared in different experiments, (2) K, SDr, and ETr were required to be identical for the RTs within each experiment, and (3) least squares estimation of parameters subject to the above constraints was applied to the means and standard deviations simultaneously.

Computation was accomplished in two steps: Model predictions were generated as a function of parameters and stored on a retrieval device, and then least squares fits at points interpolated at $1 / 3 \log _{2}$ (duration) intervals of predicted/observed means, and predicted/observed standard deviations were cal- 
Table 3

Parameter Estimates $(\mathrm{L}=.009375 \mathrm{fL})$

\begin{tabular}{|c|c|c|c|c|c|c|c|}
\hline Subject & Experiment & Intensity (l) & $\hat{\mathbf{K}}$ & $\hat{\mathbf{N}}_{\ell}$ & $\hat{r}_{i}^{-1}$ & ETTR & SD̂R \\
\hline \multirow[t]{3}{*}{1} & 4 & $\begin{array}{l}\mathrm{L} \\
\mathrm{L} / 2 \\
\mathrm{~L} / 4\end{array}$ & 13 & $\begin{array}{l}13 \\
13 \\
13\end{array}$ & $\begin{array}{l}33.0 \\
43.5 \\
54.5\end{array}$ & 183.0 & 9.0 \\
\hline & 5 & $\begin{array}{l}L / 4 \\
L / 8 \\
L / 16\end{array}$ & 10 & $\begin{array}{l}13 \\
11 \\
10\end{array}$ & $\begin{array}{l}54.5 \\
60.0 \\
68.5\end{array}$ & 210.5 & 7.0 \\
\hline & 6 & $\begin{array}{l}L / 16 \\
L / 32 \\
L / 64\end{array}$ & 9 & $\begin{array}{r}10 \\
10 \\
8\end{array}$ & $\begin{array}{l}68.5 \\
73.0 \\
90.0\end{array}$ & 230.0 & 9.0 \\
\hline \multirow[t]{3}{*}{2} & 4 & $\begin{array}{l}\mathrm{L} \\
\mathrm{L} / 2 \\
\mathrm{~L} / 4\end{array}$ & 11 & $\begin{array}{l}11 \\
11 \\
11\end{array}$ & $\begin{array}{l}42.5 \\
52.0 \\
68.0\end{array}$ & 231.0 & 10.0 \\
\hline & 5 & $\begin{array}{l}L / 4 \\
L / 8 \\
L / 16\end{array}$ & 9 & $\begin{array}{r}11 \\
9 \\
9\end{array}$ & $\begin{array}{l}68.0 \\
63.0 \\
80.0\end{array}$ & 254.0 & 10.0 \\
\hline & 6 & $\begin{array}{l}L / 16 \\
L / 32 \\
L / 64\end{array}$ & 8 & $\begin{array}{l}9 \\
8 \\
7\end{array}$ & $\begin{array}{r}80.0 \\
90.0 \\
114.0\end{array}$ & 287.1 & 7.0 \\
\hline
\end{tabular}

culated simultaneously. For the Poisson counting model, the number of free parameters for a single subject were: 3 values of $\mathrm{K}$ (one per experiment), 3 values of ETr (one per experiment), 3 values of SDr (one per experiment), 7 values of $\mathrm{N}_{l}$ (one per intensity), and 7 values of $r_{l}$ (one per intensity)-or a total of 23 free parameters. The number of data points for a single subject were 8 durations by 3 intensities by 3 experiments for both means and standard deviations, a total of 144 . Therefore, the data-point/free-parameter ratio is $144 / 23$, or 6.26 .

\section{Results of Parameter Estimates}

The magnitude of $\hat{N}_{\ell}$ varied from 7 to 13 with stimulus intensity, $\ell$, in the direction expected (cf. Table 3), monotonically nondecreasing with $\ell$. The estimated interarrival time between successive pulses for a single process, $\hat{\mathbf{r}}_{\ell}{ }^{-1}$, generally increased from about $35 \mathrm{msec}$ with decreasing light intensity up to a maximum of $114 \mathrm{msec}$ for $\mathrm{S} 2$. These values are consistent with similar estimates obtained from the TI-ED model (Hildreth, 1973), considering the lower stimulus intensities used in these experiments. (It can, in fact, be shown that the TI-ED model is a special case of this Poisson counting model, with $\mathbf{N}$ and $K=\infty$.) $\hat{K}$, the estimated number of pulses required for detection, varied from 8 to 13 with average stimulus intensity, becoming lower as average light intensity decreased in later experiments. Although $\hat{\mathrm{K}}$ and $\hat{\mathrm{N}}_{\ell}$ are similar in value, ${ }^{4}$ departure from similarity occurs when the model is more strictly tested by the larger RT variances and differences observed in the later experiments. Figures 6-11 show the goodness of fit of the model using parameter estimates of Table 3. Note that SDs and expectations were fitted simultaneously to observed data points. Reasonably good fits were obtained, considering that both means and standard deviations were predicted. The fit of RT SDs was observed to be extremely sensitive to the magnitude of both $K$ and $N_{\ell}$, with but a 2 -unit change greatly affecting predicted val-

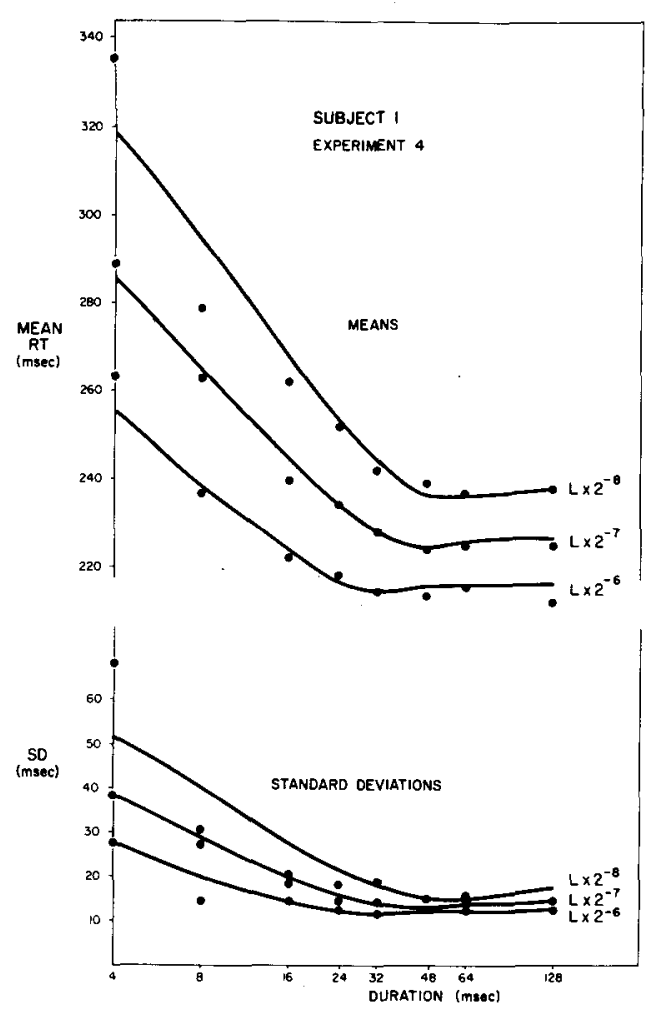

Figure 6. Goodness of fit of the Poisson counting model to observed means and standard deviations of RTs using parameter estimates of Table 3 . Least squares fits of means and variances were made simultaneously as a function of model parameters. Solid lines represent model predictions and dots observed data points. 


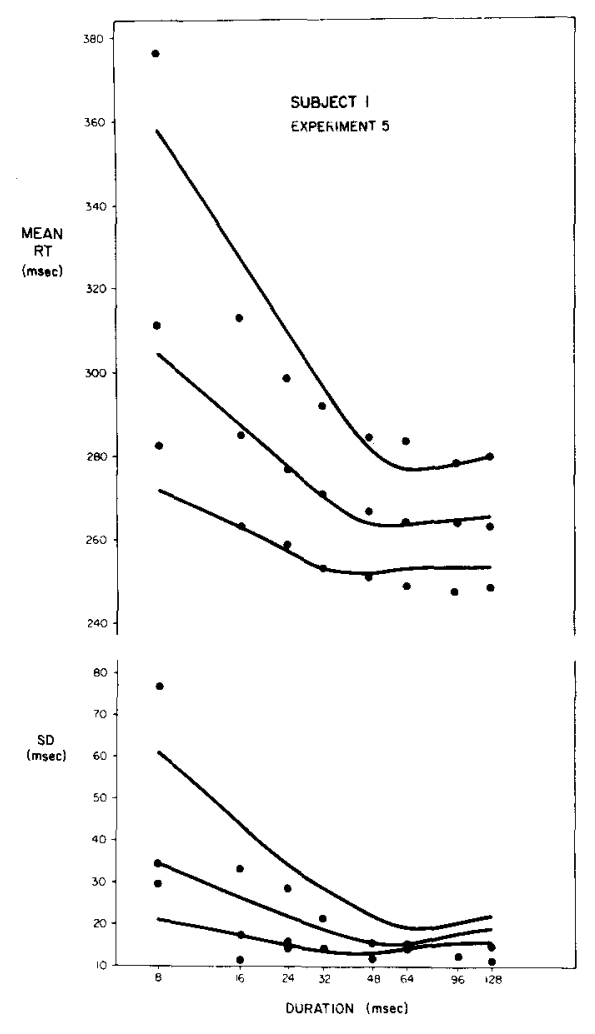

Figure 7. See caption for Figure 6.

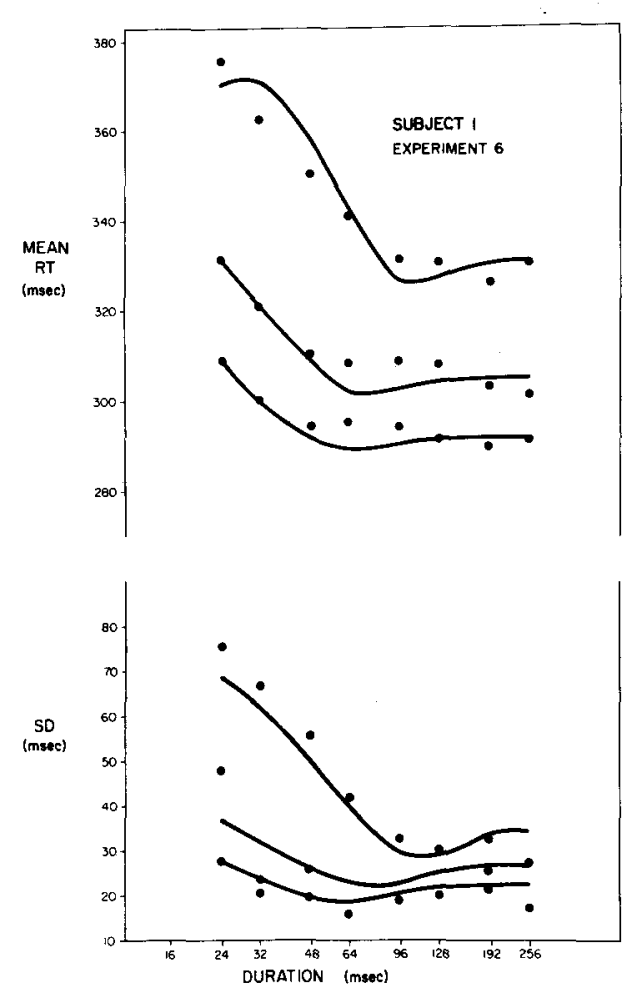

Figure 8. See caption for Figure 6.

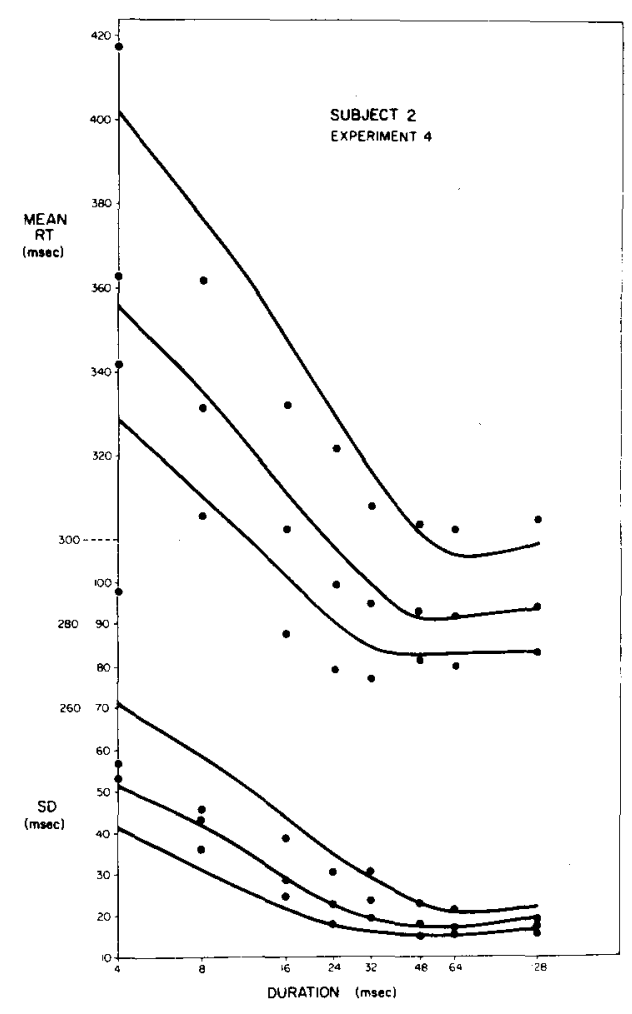

Figure 9. See caption for Figure 6.

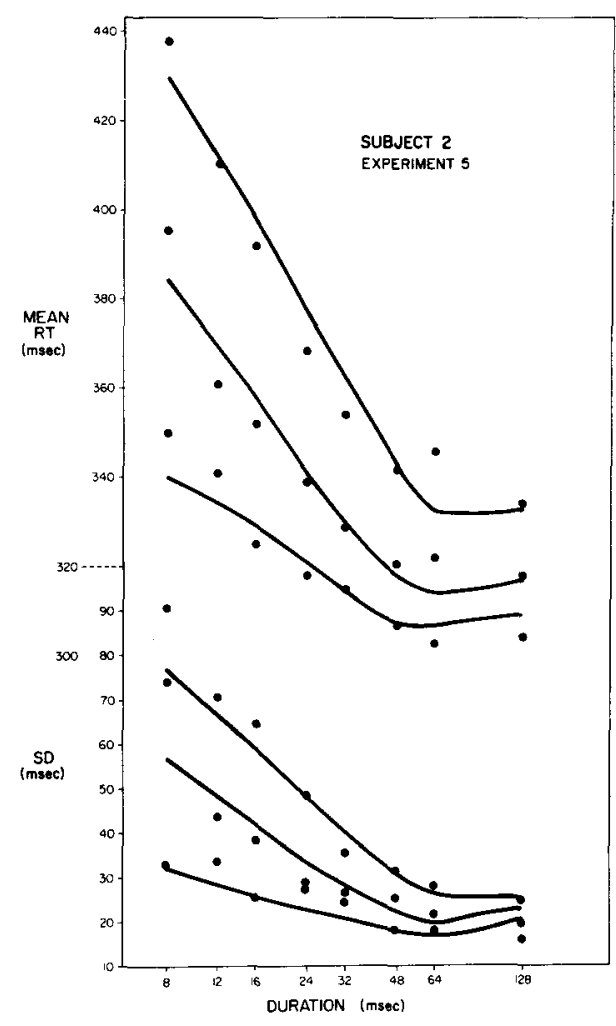

Figure 10. See caption for Figure 6. 


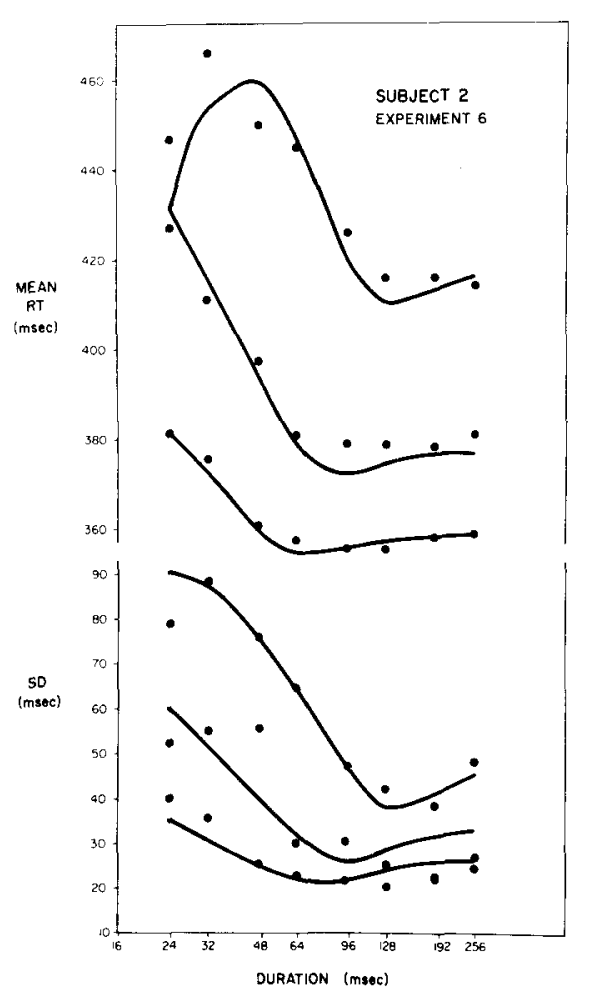

Figure 11. See caption for Figure 6.

ues. Means, on the other hand, were less sensitive to their magnitude and more sensitive to their ratio. One rather surprising result was the higher predicted means for the highest durations (Broca-Sulzer effect). This prediction of the model implies that after a certain optimum duration, light offset would actually lead to faster mean RTs than continued stimulation.

\section{Broca-Sulzer Effect}

Figures 6-11 show mean RT predicted values which rise after the critical duration. Observed mean values also rise in several experiments, showing the Broca-Sulzer effect for RT.

The Poisson counting model's prediction of the Broca-Sulzer effect is due to the fact that in the predicted curve, the mean of the truncated gamma function rises more slowly than the mean of the Poisson portion drops. The magnitude of the predicted Broca-Sulzer effect depends primarily on the last term of the equation

$$
\int_{0}^{\mathrm{d}} \mathrm{tf}_{\mathrm{k}, \ell}(\mathrm{t}) \mathrm{dt}=\frac{\mathrm{K}}{\mathrm{Nr}} \int_{0}^{\mathrm{d}} \mathrm{f}_{\mathrm{k}, \ell}(\mathrm{t}) \mathrm{dt}-\frac{\mathrm{K}}{\mathrm{Nr}} \mathrm{e}^{-\mathrm{Nrd}} \frac{(\mathrm{Nrd})^{\mathrm{K}}}{\mathrm{K} !}
$$

The Broca-Sulzer effect prediction for RT is confirmed by computation of both the mean RT function (Equation 5) and its derivative, $\frac{\partial}{\partial d} \operatorname{RT}(\mathrm{d}, \ell)$

$$
\begin{gathered}
=\int_{d}^{\infty} f_{k, \ell}(t) d t-\left[f_{k, \ell}(d)+N r \int_{d}^{\infty} f_{k, \ell}(t) d t\right] \\
\times \sum_{i=K-N}^{K-1} e^{-N r d} \frac{(N r d)}{i !} \sum_{j=1}^{K-i} \frac{1}{(N-j+1) r} .
\end{gathered}
$$

The latter is initially negative for values of $d$ less than the critical duration, then positive shortly after, and finally drops to zero as $\mathrm{d}$ approaches $\infty$.

Table 4 shows values of Equation 12 as a function of $\mathrm{d}$ for S1 E4, luminance $3(\mathrm{~K}=13, \mathrm{~N}=13$, and $1 / \mathrm{r}=54.5$ ).

Thus, the Poisson counting model predicts a BrocaSulzer effect for RT due to the nature of the stochastic process involved in the mechanism of detection.

\section{Prediction of Detection Probability}

As a stochastic model, the Poisson counting model predicts miss probabilities as well as RT statistics. If the sum of pulses counted by the time of light offset, $X_{d, \ell}$, plus the $N_{l}$ more remaining in each Poisson process is still less than $K$, the criterion, detection will not occur. Since

$$
P(\text { miss })=P\left(X_{d, \ell}<K-N_{\ell}\right) \text {, }
$$

where $\mathrm{X}_{\mathrm{d}, \ell}$ has a Poisson distribution, $\mathbf{P}\left(\mathrm{X}_{\mathrm{d}, \ell}<0\right)$ $=0$, so the only cases where misses are predicted are

Table 4

Slope of Predicted RT Function for S1 E4 Luminance 3

\begin{tabular}{rr}
\hline Duration & Slope \\
\hline 1 & -37.4 \\
6 & -26.7 \\
11 & -20.0 \\
16 & -15.4 \\
21 & -11.9 \\
26 & -9.2 \\
31 & -6.9 \\
36 & -4.9 \\
41 & -3.2 \\
46 & -1.9 \\
51 & -.9 \\
56 & -.4 \\
61 & -.1 \\
66 & +.04 \\
71 & +.07 \\
76 & +.06 \\
81 & +.04 \\
86 & +.03 \\
91 & +.02 \\
96 & +.01 \\
101 & +.01 \\
106 & +.00 \\
111 & +.00 \\
\hline
\end{tabular}

Note-Parameters $K=13, N=13, \frac{1}{r}=54.5$. 
Table 5

Predicted and Observed Miss Probabilities

\begin{tabular}{|c|c|c|c|c|c|c|c|c|c|}
\hline & & \multicolumn{8}{|c|}{ Duration } \\
\hline & & 24 & 32 & 48 & 64 & 96 & 128 & 192 & 256 \\
\hline S1 E6 (L3) & $\begin{array}{l}\text { Observed } \\
\text { Predicted }\end{array}$ & $\begin{array}{l}.27 \\
.12\end{array}$ & $\begin{array}{l}.18 \\
.06\end{array}$ & $\begin{array}{l}.03 \\
.01\end{array}$ & $\begin{array}{l}.03 \\
.00\end{array}$ & $\begin{array}{l}.00 \\
.00\end{array}$ & $\begin{array}{l}.00 \\
.00\end{array}$ & $\begin{array}{l}.00 \\
.00\end{array}$ & $\begin{array}{l}.00 \\
.00\end{array}$ \\
\hline S2 E6 (L3) & $\begin{array}{l}\text { Observed } \\
\text { Predicted }\end{array}$ & $\begin{array}{l}.54 \\
.23\end{array}$ & $\begin{array}{l}.42 \\
.14\end{array}$ & $\begin{array}{l}.17 \\
.05\end{array}$ & $\begin{array}{l}.08 \\
.00\end{array}$ & $\begin{array}{l}.00 \\
.00\end{array}$ & $\begin{array}{l}.00 \\
.00\end{array}$ & $\begin{array}{l}.00 \\
.00\end{array}$ & $\begin{array}{l}.00 \\
.00\end{array}$ \\
\hline
\end{tabular}

Note-All other predicted miss probabilities were zero.

when the estimated criterion $K$ exceeds $N_{l}$, the estimated number of Poisson processes. Table 3 shows that this happens only for Experiment 6 at the lowest intensity for both subjects. For $\mathrm{S} 1, \mathrm{~K}=9, \mathrm{~N}_{\ell}=8$, and $1 / r_{\ell}=90$. For $\mathrm{S} 2, \mathrm{~K}=8, \mathrm{~N}_{\ell}=7$, and $1 / \mathrm{r}_{\ell}=$ 114. Since for both subjects

$$
\begin{aligned}
\mathrm{P}\left(\mathrm{X}_{\mathrm{d}, \ell}<\mathrm{K}-\mathrm{N}\right) & =\mathrm{P}\left(\mathrm{X}_{\mathrm{d}, \ell}<1\right) \\
& =\mathrm{P}\left(\mathrm{X}_{\mathrm{d}, \ell}=0\right)=\mathrm{e}^{-\mathrm{N}_{\ell} \mathrm{r}_{\ell} \mathrm{d}},
\end{aligned}
$$

S1's prediction is the curve $\exp (-8 \mathrm{~d} / 90)$ and that for $\mathrm{S} 2$ is $\exp (-7 \mathrm{~d} / 114)$. Table 5 shows the observed and predicted miss probabilities. These clearly are not the best fit one might hope for. It is gratifying, however, to see the predictions qualitatively approaching observed miss proportions, considering that no detection data was used for parameter estimation; the detection probability prediction is solely from RT data.

\section{SUMMARY}

The twofold purpose of this study was to (1) determine whether Bloch's law holds for reaction times to light stimuli near threshold, and (2) provide a stochastic basis for the regular results obtained both from the experiments reported here (4-6) and from Hildreth (1973) (1-3). Bloch's law was found to be supported for both means and standard deviations of Experiments 4 and 5, but not of Experiment 6.

A stochastic model, the Poisson counting model, was advanced, which predicted RT standard deviations as well as means. In addition, the model predicted a Broca-Sulzer effect for mean RTs, which was observed in the lowest intensity experiments reported here. Finally, the increase by a constant amount of mean RTs to identical stimuli, when included in a lower intensity stimulus set, was attributed to both a variation in perceptual criterion and an increase in residual $\mathrm{RT}$.

\section{REFERENCES}

Bernstein, I. H., Futch, D. G., \& Schurman, D. L. Some exposure duration effects in simple reaction time. Journal of Experimental Psychology, 1973, 97, 317-322.

Bruder, G. E., \& Kietzman, M. L. Visual temporal integration for threshold signal detectability and RT measures. Perception \& Psychophysics, 1973, 13, 293-300.

Hildreth, J. D. Bloch's law and a temporal integration model for simple reaction time to light. Perception \& Psychophysics, $1973,14,421-432$.

Luce, R. D., \& Green, D. M. A neural timing theory for response times and the psychophysics of intensity. Psychological Review, 1972, 79, 14-57.

Standing, L. G., Dodwell, P. C., \& Lang, D. Dark adaptation and the Pulfrich effect. Perception \& Psychophysics, 1968, 4, 118-120.

\section{NOTES}

1. Sessions discarded were identified by excessive misses and/or false alarms, unusually high means, and variances. None were discarded for Experiment 4, one was discarded for Experiment 5 (S1) and two for Experiment 6 (S2).

2. Stimulus intensity was rechecked at the beginning of each experiment for each subject or when required by lamp failure.

3. Thus we have simplified the model to a noise-free model. Responses to catch trials could be predicted if it were assumed that $N_{\ell}$ for background illumination or noise were nonzero. This appears to be a natural generalization of the model, since it is obvious that background light is present and false alarms to catch trials, as well as misses, do occur.

4. Since $K$ is the subject's criterion for detection, and $N_{l}$ is the number of Poisson processes, there is no a priori relationship between $\mathbf{K}$ and $\mathbf{N}$. In model parameter estimates, however, the higher intensity experiments lead to estimates where $\hat{\mathrm{K}}=\hat{\mathrm{N}}_{\mathrm{l}}$, and the lowest intensity experiments lead to estimates where $\mathbf{K}$ exceeds $\mathrm{N}_{1}$ and the detection probability is substantially less than 1.0. The detection probability is 1.0 whenever $\mathrm{K}$ is less than or equal to $N_{\ell}$. Still, the possibility exists that the criterion $K$ could be set by the subject to be less than $N_{l}$, as is seen in some estimates of Table 3. The model predicts faster responses in such cases, with a shallower mean RT slope and much shallower variance slope. Since this relationship between parameters is not seen for the higher intensity experiments, it may somehow be optimal to set $\mathrm{K}=\mathbf{N}_{l}$, perhaps because this leads to the fewest false alarms from background illumination or noise (see footnote 3 ).

(Received for publication March 21, 1979; revision accepted June 13, 1979.) 